\title{
A new and efficient approach to time-dependent density-functional perturbation theory for optical spectroscopy
}

\author{
Brent Walker, ${ }^{1,2, \text { * }}$ A. Marco Saitta, ${ }^{3}$ Ralph Gebauer, ${ }^{1,4}$ and Stefano Baroni ${ }^{5,4, \text { 由 }}$ \\ ${ }^{1}$ ICTP - The Abdus Salam International Centre for Theoretical Physics, Strada Costiera 11, I-34014 Trieste, Italy \\ ${ }^{2}$ CNR-INFM, DEMOCRITOS National Simulation Center, Trieste, Italy \\ ${ }^{3}$ Physique des Milieux Condensés, Université Pierre et Marie Curie, Paris, France \\ ${ }^{4}$ INFM - DEMOCRITOS National Simulation Center, Trieste, Italy \\ ${ }^{5}$ SISSA - Scuola Internazionale Superiore di Studi Avanzati, Via Beirut 2-4, I-34014 Trieste, Italy
}

(Dated: October 8, 2018)

\begin{abstract}
Using a super-operator formulation of linearized time-dependent density-functional theory, the dynamical polarizability of a system of interacting electrons is given a matrix continued-fraction representation whose coefficients can be obtained from the non-symmetric block-Lanczos method. The resulting algorithm allows for the calculation of the full spectrum of a system with a computational workload which is only a few times larger than that needed for static polarizabilities within time-independent density-functional perturbation theory. The method is demonstrated with the calculation of the spectrum of benzene, and prospects for its application to the large-scale calculation of optical spectra are discussed.

PACS numbers: 31.15.-p 71.15.Qe 31.15.Ew 71.15.Mb 33.20.Lg
\end{abstract}

The past two decades have witnessed the tremendous success of density-functional theory (DFT) [1, 2] in describing and predicting various properties of systems of interacting electrons, ranging from atoms and molecules to solids and liquids. In its original formulation, DFT only applies to the electronic ground state. This limitation was lifted by Runge and Gross (RG) [3] who generalized DFT to time-dependent systems. According to the RG theorem, for any given $(t=0)$ initial state of the many-body system, the external, generally time-dependent, potential acting on it is uniquely determined by the time evolution of the one-electron density, $n(\mathbf{r}, t)$, for $t>0$. Based on this theorem, it is possible to formally establish a time-dependent Kohn-Sham (KS) equation from which various one-particle properties of the system can be obtained as functions of time. The resulting theoretical framework is usually referred to as time-dependent densityfunctional theory (TDDFT). Unfortunately, little is known about the exchange-correlation (XC) potential in ordinary DFT, and even less is known about it in the time-dependent case. Most of the existing applications of TDDFT are based on the adiabatic local density (ALDA) or adiabatic generalized gradient approximations, which amount to using the same functional dependence of the XC potential upon density as in the static case. In spite of the crudeness of these approximations, the optical spectra calculated using them is in some cases almost as accurate as those obtained from more computationally demanding many-body approaches [4]. TDDFT is in principle an exact theory. Progress in understanding and characterizing the $\mathrm{XC}$ functional will thus substantially increase the predictive power of TDDFT while keeping its computational requirements at a significantly more modest level than methods based on many-body perturbation theory.

Linearization of TDDFT with respect to the strength of some external perturbation to an otherwise time-independent system leads to a non-Hermitean eigenvalue problem whose eigenvalues are excitation energies of the system, and whose eigenvectors can be used to calculate appropriate oscillator strengths [5]. Not surprisingly, this eigenvalue problem has the same structure as that arising from time-dependent Hartree-Fock theory [6], and the dimension of the resulting matrix (the Liouvillian) is thus twice the product of the number of occupied states, $N_{v}$, with the number of empty states. The diagonalization of such a large matrix can be accomplished using iterative techniques, often in conjunction with the so-called Tamm-Dancoff approximation (TDA) which amounts to enforcing Hermiticity by neglecting the anti-Hermitean component of the Liouvillian [7]. Many of the existing molecular applications of TDDFT have been performed within such a framework which suffers however from two major limitations. The first is that many individual eigenvalues/eigenvectors must be calculated in order to model the spectral properties in any given frequency range, and the number of such eigenpairs increases with the size of the system. The second limitation is that a straightforward implementation of this method preliminarily requires the full diagonalization of the unperturbed Kohn-Sham Hamiltonian, a task which may become prohibitive for large systems and/or when the one-electron basis set is very large (as may be the case with plane waves or real-space grids). The first of these problems is sometimes dealt with by directly calculating the relevant response function(s), rather than individual excitations [4]. The price paid in this case is the manipulation (inversion, multiplication) of large matrices for any individual frequency, a task which may again be impossible for large systems/basis sets. Even so, the full diagonalization of the unperturbed KS Hamiltonian cannot be avoided.

An alternative approach to TDDFT, first proposed by Yabana and Bertsch [8], avoids diagonalization altogether. In this approach, the TDDFT KS equations are solved in the time domain and susceptibilities are obtained by Fourier analyzing the response of the system to appropriate perturbations in the linear regime. This scheme has the same computational 
complexity as standard time-independent iterative methods in DFT. For this reason, real-time methods have recently gained popularity in conjunction with the use of real-space grids [9], and a similar success should be expected using plane-wave (PW) basis sets. The main limitation of these methods is that the time step needed for a stable integration of the TDDFT KS equations is small (of the order of $10^{-3}$ fs in typical pseudopotential applications) and decreases as the number of PW's (or real-space grid points) increases.

In this Letter we propose a novel way to calculate optical spectra in the frequency domain - thus avoiding any explicit integration of the TDDFT KS equations-which does not require any diagonalization (of either the unperturbed KS Hamiltonian, or the TDDFT Liouvillian), nor any timeconsuming matrix operations. To this end, we first express a generalized susceptibility as a matrix element of the resolvent of the Liouvillian super-operator, defined in some appropriate operator space, between suitable operator states. This matrix element is then evaluated using a Lanczos continued-fraction technique. Each link of the Lanczos chain-that is calculated once for all frequencies-requires a number of floating-point operations which is only a few times larger than that needed by a single step of the iterative calculation of a static polarizability within time-independent density-functional perturbation theory (DFPT) [10, 11].

Let us first consider an external perturbation whose Fourier transform will be indicated by $\lambda(\omega) \tilde{V}^{\prime}{ }_{\text {ext }}(\mathbf{r}, \omega)$, where $\lambda(\omega)$ conventionally indicates the strength of the perturbation. Let $\varphi_{v}^{\circ}(\mathbf{r})$ and $\epsilon_{v}$ be the ground-state valence KS orbitals and energies, and $\varphi_{v}(\mathbf{r}, t)$ the solution of the TDDFT KS equation with the initial condition $\varphi_{v}(\mathbf{r}, 0)=\varphi_{v}^{\circ}(\mathbf{r})$. We define the orbital response functions as $\varphi_{v}^{\prime}(\mathbf{r}, t)=\mathrm{e}^{i \epsilon_{v} t}\left(\varphi_{v}(\mathbf{r}, t)-\varphi_{v}^{\circ}(\mathbf{r})\right)$, and indicate their Fourier transforms as $\varphi_{v}^{+}(\mathbf{r})=\tilde{\varphi}_{v}^{\prime}(\mathbf{r}, \omega)$, and $\varphi_{v}^{-}(\mathbf{r})=\tilde{\varphi}_{v}^{\prime *}(\mathbf{r},-\omega)$. The $\varphi_{v}^{ \pm}$orbitals can be chosen to be orthogonal to the Kohn-Sham occupied manifold, and they can be easily shown to satisfy the coupled linear equations:

$$
\begin{aligned}
\omega \varphi_{v}^{+}(\mathbf{r}) & =\left(\hat{H}_{K S}^{\circ}-\epsilon_{v}\right) \varphi_{v}^{+}(\mathbf{r})+\hat{P}_{c} \tilde{V}^{\prime}(\mathbf{r}, \omega) \varphi_{v}^{\circ}(\mathbf{r}), \\
-\omega \varphi_{v}^{-}(\mathbf{r}) & =\left(\hat{H}_{K S}^{\circ}-\epsilon_{v}\right) \varphi_{v}^{-}(\mathbf{r})+\hat{P}_{c} \tilde{V}^{\prime}(\mathbf{r}, \omega) \varphi_{v}^{\circ}(\mathbf{r}),(1)
\end{aligned}
$$

where $\hat{H}_{K S}^{\circ}$ is the ground-state KS Hamiltonian, $\hat{P}_{c}$ is the projector onto the KS empty-state manifold, $\tilde{V}^{\prime}(\mathbf{r}, \omega)=$ $\tilde{V}_{\text {ext }}^{\prime}(\mathbf{r}, \omega)+\int \kappa\left(\mathbf{r}, \mathbf{r}^{\prime}\right) \tilde{n}^{\prime}\left(\mathbf{r}^{\prime}, \omega\right) d \mathbf{r}^{\prime}$ is the perturbing KS potential, $\tilde{n}^{\prime}(\mathbf{r}, \omega)=\sum_{v=1}^{N_{v}} \varphi_{v}^{\circ}(\mathbf{r})\left(\varphi_{v}^{+}(\mathbf{r})+\varphi_{v}^{-}(\mathbf{r})\right)$ is the linear variation of the electron density, and $\kappa\left(\mathbf{r}, \mathbf{r}^{\prime}\right)=\frac{e^{2}}{\left|\mathbf{r}-\mathbf{r}^{\prime}\right|}+$ $\left.\frac{\delta V_{X C}(\mathbf{r})}{\delta n\left(\mathbf{r}^{\prime}\right)}\right|_{n=n^{\circ}}$ is the Hartree plus XC kernel. In Eq. (1) use has been made of the fact that the perturbation is real $\left[\tilde{V}^{\prime *}(\mathbf{r},-\omega)=\tilde{V}^{\prime}(\mathbf{r}, \omega)\right]$, while in the expression for $\tilde{n}^{\prime}$, the $\varphi^{\circ}$ 's are assumed to be real due to time-reversal invariance. Following Refs. 12 and 13 , we now consider the supervector $|X\rangle \equiv|\mathbf{x}, \mathbf{y}\rangle$, where $\mathbf{x}$ and $\mathbf{y}$ are batches of orbitals, $\mathbf{x}=\left\{x_{v}(\mathbf{r})\right\}, \mathbf{y}=\left\{y_{v}(\mathbf{r})\right\}$, defined as: $x_{v}(\mathbf{r})=\frac{1}{2}\left[\varphi_{v}^{+}(\mathbf{r})+\right.$ $\left.\varphi_{v}^{-}(\mathbf{r})\right]$, and $y_{v}(\mathbf{r})=\frac{1}{2}\left[\varphi_{v}^{+}(\mathbf{r})-\varphi_{v}^{-}(\mathbf{r})\right]$. The fundamental
TDDFT equations, Eq. (1), can then be formally written as:

$$
(\omega-\mathcal{L})|\mathbf{x}, \mathbf{y}\rangle=|0, \mathbf{v}\rangle
$$

where the batch $\mathbf{v}$ is defined as $\mathbf{v}=\left\{\hat{P}_{c} \tilde{V}_{\text {ext }}^{\prime}(\mathbf{r}, \omega) \varphi_{v}^{\circ}(\mathbf{r})\right\}$, the Liouvillian super-operator $\mathcal{L}$ is defined as: $\mathcal{L}|\mathbf{x}, \mathbf{y}\rangle=\mid \mathcal{D}$. $\mathbf{y},(\mathcal{D}+\mathcal{K}) \cdot \mathbf{x}\rangle$, and $\mathcal{D}$ and $\mathcal{K}$ are Hermitean operators acting on batches of response orbitals, $\mathbf{u}=\left\{u_{v}(\mathbf{r})\right\}$, as:

$$
\begin{aligned}
& \mathcal{D} \cdot \mathbf{u}=\left\{\left(\hat{H}_{K S}^{\circ}-\epsilon_{v}\right) u_{v}(\mathbf{r})\right\} \\
& \mathcal{K} \cdot \mathbf{u}=\left\{\varphi_{v}^{\circ}(\mathbf{r}) \sum_{v^{\prime}} \int \kappa\left(\mathbf{r}, \mathbf{r}^{\prime}\right) \varphi_{v^{\prime}}^{\circ}\left(\mathbf{r}^{\prime}\right) u_{v^{\prime}}\left(\mathbf{r}^{\prime}\right) d \mathbf{r}^{\prime}\right\} .
\end{aligned}
$$

Eq. (2) gives the response of the system to the external perturbation, as a function of frequency. When $V_{e x t}^{\prime}=0$, this equation reduces to a (non-Hermitean) eigenvalue problem whose eigenvectors describe the free oscillations of the system corresponding to electronic excitations. This is essentially equivalent to Casida's formulation of TDDFT [5].

In practice, one is seldom interested in the response of the system to the most general perturbation, or in individual excitation energies, but just in the frequency-dependent response of some specific property to some specific perturbation. Let us consider an observable, $\hat{A}$, whose time-dependent linear response is given by: $A(t)=2 \operatorname{Re} \sum_{v}\left\langle\varphi_{v}^{\circ} \mid \varphi_{v}^{\prime}(t)\right\rangle$. Assuming that $\hat{A}$ is time-reversal invariant, the Fourier transform of $A(t)$ can be written as: $\tilde{A}(\omega)=\sum_{v}\left(\left\langle\varphi_{v}^{\circ}|\hat{A}| \varphi_{v}^{+}\right\rangle+\left\langle\varphi_{v}^{\circ}|\hat{A}| \varphi_{v}^{-}\right\rangle\right) \equiv$ $2\langle\mathbf{a}, 0 \mid \mathbf{x}, \mathbf{y}\rangle$, where $\mathbf{a}=\left\{\hat{A} \varphi_{v}^{\circ}\right\}$. A generalized susceptibility, $\chi_{A V}(\omega)$, can be defined as the derivative of $\tilde{A}(\omega)$ with respect to the strength of the perturbation, $\lambda(\omega)$. Using Eq. (2) and the linearity of $\tilde{A}(\omega)$ with respect to $\lambda(\omega)$, the susceptibility can be written as:

$$
\chi_{A V}(\omega)=2\left\langle\mathbf{a}, 0\left|(\omega-\mathcal{L})^{-1}\right| 0, \mathbf{v}\right\rangle .
$$

The results obtained so far and embodied in Eq. (5) can be summarized by saying that within TDDFT any generalized susceptibility can be expressed as an appropriate offdiagonal matrix element of the resolvent of the Liouvillian super-operator. In the following we show how such a matrix element can be conveniently calculated using a matrix continued fraction resulting from the non-Hermitean block-Lanczos algorithm (NHBLA) [14].

Let us define a block, $|\mathbf{Q}\rangle$, as a pair of orthogonal supervectors: $|\mathbf{Q}\rangle=\left\{\left|Q_{1}\right\rangle,\left|Q_{2}\right\rangle\right\}$. The scalar product between two blocks, $=\langle\mathbf{P} \mid \mathbf{Q}\rangle$ is defined as the $2 \times 2$ matrix: $s_{i j}=$ $\left\langle P_{i} \mid Q_{j}\right\rangle$, and the action of a super-operator on a block is defined as the block whose elements are the result of the action of the super-operator on each of the two elements of the original block: $\mathcal{L}\left|\left\{Q_{1}, Q_{2}\right\}\right\rangle \equiv\left|\left\{\mathcal{L} Q_{1}, \mathcal{L} Q_{2}\right\}\right\rangle$. Given two starting blocks, $\left|\mathbf{Q}^{1}\right\rangle$ and $\left|\mathbf{P}^{1}\right\rangle$ such that $\left\langle P_{i}^{1} \mid Q_{j}^{1}\right\rangle=\delta_{i j}$, the NHBLA allows one to generate a sequence of block pairs, $\left\{\left|\mathbf{Q}^{n}\right\rangle,\left|\mathbf{P}^{n}\right\rangle\right\}$, such that: $\left\langle P_{i}^{n} \mid Q_{j}^{m}\right\rangle=\delta_{m n} \delta_{i j}$, and $\mathcal{L}=$ $\sum_{n m, i j} T_{i j}^{m n}\left|Q_{i}^{m}\right\rangle\left\langle P_{j}^{n}\right|$, where: $T_{i j}^{n m}=\left\langle P_{i}^{n}|\mathcal{L}| Q_{j}^{m}\right\rangle=$ $a_{i j}^{n} \delta_{m n}+b_{i j}^{n} \delta_{m+1, n}+c_{i j}^{m} \delta_{m, n+1}$ is a block-tridiagonal matrix. Using these relations, the projection of the resolvent of 


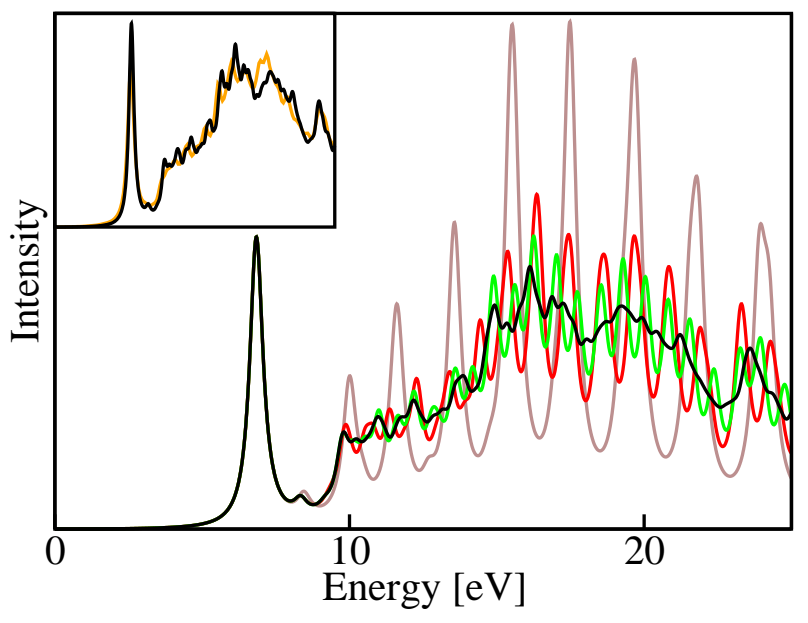

FIG. 1: Absorption spectrum of benzene calculated using the Lanczos method with different numbers of recursion steps: 1000 (plum), 2000 (red), 3000 (green), and 6000 (black). The inset compares the 6000 -step spectrum (black) with that obtained using the real-time propagation method (orange).

the Liouvillian over the starting block pair can then be easily expressed as a matrix continued fraction:

$$
\left\langle\mathbf{P}_{1}\left|(\omega-\mathcal{L})^{-1}\right| \mathbf{Q}_{1}\right\rangle=\frac{1}{\omega-\mathbf{a}_{1}+\mathbf{b}_{2} \frac{1}{\omega-\mathbf{a}_{2}+\cdots} \mathbf{c}_{2}},
$$

where the a's, b's, c's, are $2 \times 2$ matrices. If the starting block is chosen as $\left|Q_{1}^{1}\right\rangle=|\mathbf{a}, 0\rangle$, and $\left|Q_{2}^{1}\right\rangle=|0, \mathbf{v}\rangle$, the generalized susceptibility, Eq. [5), is then the $(1,2)$ matrix element of the $2 \times 2$ matrix given by Eq. (6). Without going into the details of the NHBLA, suffice it to say that its implementation does not require the explicit calculation of the Liouvillian superoperator, nor even of the unperturbed KS Hamiltonian, but just the availability of a black-box computer routine which, for any given batch of response functions, $\mathbf{u}=\left\{u_{v}(\mathbf{r})\right\}$, returns $\mathcal{D}|\mathbf{u}\rangle$ and $\mathcal{K}|\mathbf{u}\rangle$, according to Eqs. (3) and (4). Each step of the NHBLA essentially involves two calls to such a routine whose computational cost is roughly the same as that of a single iteration in a static DFPT calculation.

The theory described above was implemented on top of the PWscf plane-wave pseudopotential code which is part of the Quantum ESPRESSO distribution [15]. As a test to demonstrate our methodology, we have calculated the absorption spectrum of benzene, a system for which many excited-state calculations already exist [18, 19], some of which were performed within TDDFT [19]. The KS equations for an isolated benzene molecule were solved using periodic boundary conditions $(\mathrm{PBC})$ in a tetragonal simulation cell whose base, parallel to the carbon ring (' $x y$ ' plane), has a side length $30.0 \AA$, and with height $2 / 3$ of this; the $\mathrm{CC}$ and $\mathrm{CH}$ bond lengths were set to $1.40 \AA$ and $1.09 \AA$, respectively. A simple LDA exchange-correlation functional was adopted [16], whereas norm-conserving pseudopotentials from the PWscf table were used [17] with a PW kinetic-energy cutoff of 60.0
Ry. The PBC calculation of dipole matrix elements was performed as explained in Sec. C.2 of Ref. 11. The absorption coefficient was obtained as: $I(\omega) \propto \omega \operatorname{Im} \chi(\omega)$, where $\chi(\omega)$ is the electric dipole susceptibility, Eq. (5), calculated at complex frequencies with an imaginary part of $0.27 \mathrm{eV}$.

The convergence properties of our block-Lanczos algorithm are displayed in Fig. 11 where we report the absorption spectra of benzene as calculated for light polarized in the $x y$ plane, for different numbers of recursion steps. We see that 2000-3000 steps are sufficient to ensure convergence for energies up to $\approx 15 \mathrm{ev}$. Our most converged spectrum (corresponding to 6000 recursion steps) is then compared with that obtained using the real-time propagation method (see the inset). The agreement between the spectra obtained with these rather different methods is practically complete. Although a thorough theoretical analysis of the convergence properties of our algorithm is beyond the scope of the present letter, we would like to point out a few facts that, we believe, will deserve further attention. We first notice that, not unexpectedly, the convergence properties deteriorate with increasing frequency: the lower the frequency, the better the convergence. Second, the convergence rate of the algorithm seems to be somewhat affected by the condition number of the Liouvillian which, in turn, depends on the size of the PW basis set: the higher the PW kinetic-energy cutoff, the worse the convergence [20]. Furthermore, the numerical instabilities which are known to plague Lanczos diagonalization algorithms [14] seem to have little, if any, effect on the calculation of the resolvent matrix elements through Eq. (6). As far as we can say, Eq. (6) can be pushed as much as needed to reach any desired level of accuracy. Finally, the non-Hermitean character of the Liouvillian seems to affect somewhat the efficiency of the algorithm, as can be seen from the performance of the TDA that we examine now.

In Fig. 2we compare the experimental absorption spectrum of Ref. [21] with those obtained from our TDDFT method, with and without use of the TDA. The agreement between the calculated and experimental spectra is very good, as was already known from previous TDDFT calculations for benzene [19]. The quality of this agreement is due to the nature of the molecular orbitals involved in the transitions which dominate the low-lying part of the spectrum $\left(\pi, \pi^{*}\right.$, and, to a lesser extent, $\sigma$ ), which are little affected by the wrong asymptotic behavior of the ALDA XC potential. The absorption spectra reported in Fig. 2] though resulting from an average over different polarizations, are dominated by the $\left(\pi^{*} \leftarrow \pi\right){ }^{1} E_{1 u}$ transition which is only allowed when the light is polarized in the plane of the molecule. This transition is mainly responsible for the first strong absorption peak experimentally found at $6.94 \mathrm{eV}$, and predicted by TDDFT at $6.83 \mathrm{eV}$. It is interesting to notice that the $z$ component of the spectrum displays a weak peak at $6.55 \mathrm{eV}$, which is not visible in Fig. 2 (its intensity is more than 10 times smaller than the $x y$ peak), and which corresponds to a $\left(\pi^{*} \leftarrow \sigma\right){ }^{1} A_{2 u}$ transition. In the independentelectron approximation, this transition would have a higher energy than ${ }^{1} E_{1 u}$ which corresponds to the HOMO-LUMO 


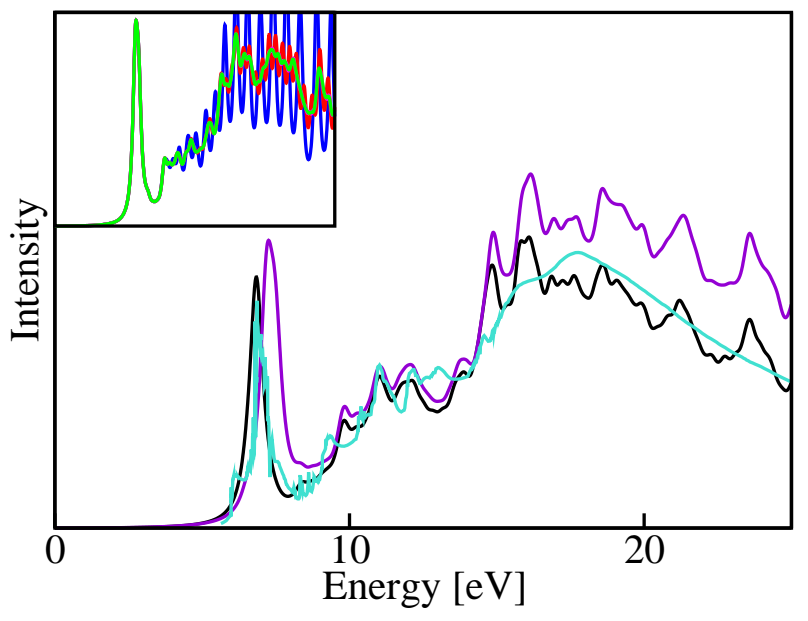

FIG. 2: Comparison between the converged Lanczos spectrum obtained with the full non-Hermitean Liouvillian (black), the TammDancoff approximation (red) and the experimental results (light blue). The inset shows the convergence of the TDA spectrum with respect to the number of recursion steps: 250 (blue), 5001000 (magenta), and 2000 (orange).

gap. The red shift of the ${ }^{1} A_{2 u}$ transition is therefore due to the effects of the electron-electron interaction which are approximately accounted for in ALDA-TDDFT. The ${ }^{1} A_{2 u}$ transition has never been detected directly in absorption experiments, but its existence (as well as its location near, possibly at a lower frequency than, the strong ${ }^{1} E_{1 u}$ transition) was inferred from Raman scattering experiments [22]. The proximity of the ${ }^{1} E_{1 u}$ and ${ }^{1} A_{2 u}$, as well as the much smaller intensity of the latter, were also confirmed by accurate coupled-cluster calculations [18d]. As far as we can tell, it is not impossible that the little shoulder observed at $6.19 \mathrm{eV}$ in the absorption spectrum [21] and attributed to a vibron-assisted ${ }^{1} B_{1 u}$ forbidden excitation, is actually due to a weak ${ }^{1} A_{2 u}$ allowed transition.

Use of the TDA does not change much the overall appearance of the spectrum, nor the positions of the peaks, the main difference regarding their intensities. It is worth noticing that the convergence of TDA calculations appears to be much faster than when using the full non-Hermitean form of the Liouvillian (see the inset of Fig. 2).

We believe that the method presented in this letter, while not touching our still serious ignorance about the form of time-dependent XC functionals, will open the way to a systematic study of systems which are too large to be treated with currently available methods. The already rather favorable numerical features of our method will be further improved either by implementing the use of ultra-soft pseudopotentials (which will allow reduction of both the size of one-electron basis sets and the condition number of the Liouvillian), and by devising optimal strategies for restarting the Lanczos chain using approximate schemes. Work is in progress along these lines.

One of us (SB) wishes to thank Y. Saad for very useful dis- cussions, as well as A. Polian for hospitality at the Université Pierre et Marie Curie, Paris, where this work was started, and R. Wentzcovitch for hospitality at the University of Minnesota, where most of this paper was written.

* Electronic address: bwalker@ictp.it

$\dagger$ Electronic address: baroni@sissa.it

[1] P. Hohenberg and W. Kohn, Phys. Rev. 136, B864 (1964).

[2] W. Kohn and L.J. Sham, Phys. Rev. 140, A1133 (1965).

[3] E. Runge and E.K.U. Gross, Phys. Rev. Lett. 52, 997 (1984).

[4] G. Onida, L. Reining, and A. Rubio, Rev. Mod. Phys. 74, 601 (2002).

[5] M.E. Casida, in Recent Advances in Density Functional Methods, Part I, edited by D.P. Chong (Singapore, World Scientific, 1995), p. 155.

[6] See. e.g. A.D. McLachlan and M.A. Ball, Rev. Mod. Phys. 36, 844 (1964).

[7] I. Tamm, J. Phys. (USSR) 9, 449 (1945); S.M. Dancoff, Phys. Rev. 78, 382 (1950); S. Hirata and M. Head-Gordon, Chem. Phys. Lett. 314, 291 (1999).

[8] K. Yabana and G.F. Bertsch, Phys. Rev. B 54, 4484 (1996).

[9] G.F. Bertsch, J.-I. Iwata, A. Rubio, and K. Yabana, Phys. Rev. B 62, 7998 (2000); M.A.L. Marques, X. Lopez, D. Varsano, A. Castro, and A. Rubio, Phys. Rev. Lett. 90, 258101 (2003).

[10] S. Baroni, P. Giannozzi, and A. Testa, Phys. Rev. Lett. 58, 1861 (1987).

[11] S. Baroni, S. de Gironcoli, A. Dal Corso, and P. Giannozzi, Rev. Mod. Phys. 73, 515 (2001).

[12] J. Hutter, J. Chem. Phys. 118, 3928 (2003).

[13] E.V. Tsiper, J. Phys. B 34, L401 (2001).

[14] Z. Bai and D. Day, Block Lanczos Methods (Section 7.9) in Z. Bai, J. Demmel, J. Dongarra, A. Ruhe, and H. van der Vorst, editors, Templates for the Solution of Algebraic Eigenvalue Problems: A Practical Guide (SIAM, Philadelphia, 2000).

[15] S. Baroni et al., http: / /www. quantum-espresso.org/. See also http: //www. pwscf. org/.

[16] J.P. Perdew and A. Zunger, Phys. Rev. B 23, 5048 (1981).

[17] http://www.pwscf.org/pseudo.htm C.pz-vbc.UPF and H.pz-vbc.UPF for carbon and hydrogen respectively.

[18] (a) J. Lorentzon, P.-A. Malmquist, M. Fulscher, and B.O. Roos, Theor. Chim. Acta, 91, 91 (1995); (b) M.J. Packer, E.K. Dalskov, T. Enevoldsen, H.J. Jensen, and J.J. Oddershede, J. Chem. Phys. 105, 5586 (1996); (c) O. Christiansen, H. Koch, A. Halkier, P. Jorgensen, T. Helgaker, and A.S. de Meras, J. Chem. Phys. 105, 6921 (1996); (d) A. Christiansen, C. Hättig, and P. Jørgensen, Spetcrochim. Acta, Part A 55, 509 (1999).

[19] N.C. Handy and D.J Tozer, J. Comp. Chem. 20, 106 (1999); K. Yabana and G.F. Bertsch, Int. J. Quant. Chem. 75, 55 (1999); H.H. Heinze, A. Görling, and N. Rösch, J. Chem. Phys. 113, 2088 (2000).

[20] The calculation of the spectrum of silane, performed with a kinetic-energy cutoff of $35 \mathrm{Ry}$, shows full convergence with a number of Lanczos recursions in between 1000 and 2000.

[21] E.E. Koch and A. Otto, Chem. Phys. Lett. 12, 476 (1972).

[22] L. Ziegler and A.C. Albrecht, J. Chem. Phys. 67, 2753 (1977); M. Ito, H. Abe, and J. Murakami, J. Chem. Phys. 69, 606 (1978). 